\title{
Fluxos de Radiação e Energia em Caatinga Preservada e Cana-de-Açúcar no Semiárido
}

\author{
Herica Fernanda de Sousa Carvalho ${ }^{1}$ id , Magna Soelma Beserra de Moura ${ }^{2}$, \\ Thieres George Freire da Silva ${ }^{3}$ \\ ${ }^{I}$ Departamento de Engenharia Agrícola, Universidade Federal do Vale do São Francisco, \\ Juazeiro, BA, Brasil. \\ ${ }^{2}$ Laboratório de Agrometeorologia, Empresa Brasileira de Pesquisa Agropecuária, \\ Petrolina, PE, Brasil. \\ ${ }^{3}$ Universidade Federal Rural de Pernambuco, Unidade Acadêmica de Serra Talhada, \\ Serra Talhada, PE, Brasil.
}

Recebido em 24 de Setembro de 2017 - Aceito em 3 de Julho de 2018

\begin{abstract}
Resumo
Objetivou-se comparar os fluxos de radiação e de energia entre uma área de caatinga preservada e um cultivo de cana-de-açúcar irrigada no Semiárido brasileiro. Para tanto, foram analisados os componentes dos balanços de radiação e de energia, os dados ambientais (umidade, temperatura do solo, e regime hídrico) e o índice de cobertura vegetal, entre junho de 2015 e maio de 2016. No centro de cada área foi instalada uma torre micrometeorológica equipada com sensores eletrônicos. Como resultados, constatou-se que as principais diferenças no balanço de radiação entre a caatinga e a cana-de-açúcar estão associadas ao albedo e a emissão de radiação de ondas longas pelas superfícies. O fluxo de calor sensível $(\mathrm{H})$ foi sempre maior na caatinga, enquanto o fluxo de calor latente (LE) foi superior na cana-de-açúcar $(\mathrm{p}<0,05)$. Na caatinga, as partições de energia para o H, LE e fluxo de calor no solo (G) foram $64 \%, 34 \%$, e $2 \%$, nessa ordem; enquanto na cana-de-açúcar, foi de $12 \%$ para o H, $85 \%$ para o LE e $3 \%$ para o G; com diferenças significativas entre as superfícies $(\mathrm{p}<0,05)$. Essas diferenças podem acarretar modificações no clima local e/ou regional, logo, devem ser consideradas nos modelos de simulação hidrológicos e climáticos.
\end{abstract}

Palavras-chave: alteração climática, balanço de energia, mudança de uso da terra.

\section{Radiation and Energy Fluxes in Preserved Caatinga and Sugarcane in Semi-Arid}

\begin{abstract}
The objective of this study was to compare the radiation and energy fluxes between an area of preserved caatinga and an irrigated sugarcane crop in the Brazilian semi-arid region. The radiation and energy components, the environmental data (soil moisture and temperature, water regime) and the vegetation cover index were analyzed between June 2015 and May 2016. In each area was installed a micrometeorological tower equipped with electronic sensors. As results, it was verified that the main differences in the radiation balance between caatinga and sugarcane are associated to the albedo and the emission of long-wave radiation on the surfaces. The sensible heat flux $(\mathrm{H})$ was always higher in the caatinga, while the latent heat flux (LE) was higher in sugarcane $(\mathrm{p}<0.05)$. In the caatinga, the energy partitions for $\mathrm{H}$, LE and soil heat flux $(\mathrm{G})$ were $64 \%, 34 \%$ and $2 \%$, in that order; while in sugarcane, it was $12 \%$ for $\mathrm{H}, 85 \%$ for $\mathrm{LE}$ and $3 \%$ for G; with significant differences between the surfaces $(\mathrm{p}<0.05)$. These differences can lead to modifications in the local and / or regional climate, so they should be considered in hydrological and climatic simulation models.
\end{abstract}

Keywords: climate change, energy balance, land use change.

Autor de correspondência: Thieres George Freire da Silva, thigeoprofissional@hotmail.com. 


\section{Introdução}

A Caatinga é um bioma exclusivamente brasileiro, que ocupa $11 \%$ do território nacional, com ampla diversidade da fauna e da flora (MMA, 2015). Apesar de sua vasta extensão territorial este bioma vem sendo modificado de forma acelerada e segundo dados do MMA (2015), o desmatamento já chega a $46 \%$ da área total.

Na região do Submédio do Vale do São Francisco, especificamente nas cidades de Petrolina-PE e Juazeiro$\mathrm{BA}$, a retirada de vegetação natural ocorreu com a expansão da atividade agrícola, sobretudo para plantio de culturas irrigadas nas últimas décadas, com destaque para diversas frutíferas e cana-de-açúcar. Esta última, já ocupa área superior a 15.132 ha (IBGE, 2014), representando extensa área de monocultivo com alta relevância socioeconômica na região.

Estudos indicam que a substituição da vegetação nativa por monocultivos altera o nível de cobertura vegetal e, logo, o balanço de radiação e energia em escala local, ocasionando mudanças na hidrologia e no clima (Spracklen et al., 2010; David et al., 2011; Lu et al., 2011; Chambers; Artaxo, 2017).

Medições micrometeorológicas em áreas de caatinga (Oliveira et al., 2006; Teixeira et al., 2008; Souza et al., 2015a,b) e de cana-de-açúcar (Silva et al., 2011) já foram conduzidas no Submédio do Vale do São Francisco, mostrando a forte sazonalidade da densidade de fluxos de energia e massa ao longo das estações do ano e em função do regime hídrico, em processo de feedback com a dinâmica vegetal.

No entanto, pesquisas conduzidas simultaneamente, de modo a inferir quais as principais alterações microclimáticas promovidas pela substituição da caatinga por áreas de cultivo de cana-de-açúcar irrigada ainda são incipientes. Desta forma, objetivou-se comparar os fluxos de radiação e de energia entre uma área de caatinga preservada e um cultivo da cana-de-açúcar irrigada em ambiente Semiárido brasileiro, com vistas à compreensão dos impactos da substituição da vegetação nativa por áreas agrícolas.

\section{Material e Métodos}

$\mathrm{O}$ experimento foi realizado simultaneamente entre junho de 2015 e maio de 2016, em dois sítios experimentais: um de caatinga preservada e outro de cana-deaçúcar irrigada, localizados no Semiárido brasileiro, Submédio do Vale São Francisco (Fig. 1). A condição climática segundo a classificação de Köopen, é do tipo BSwh', tropical semiárido, com temperatura do ar variando entre a mínima de $20,7^{\circ} \mathrm{C}$ e a máxima de $32,1^{\circ} \mathrm{C}$, com média de
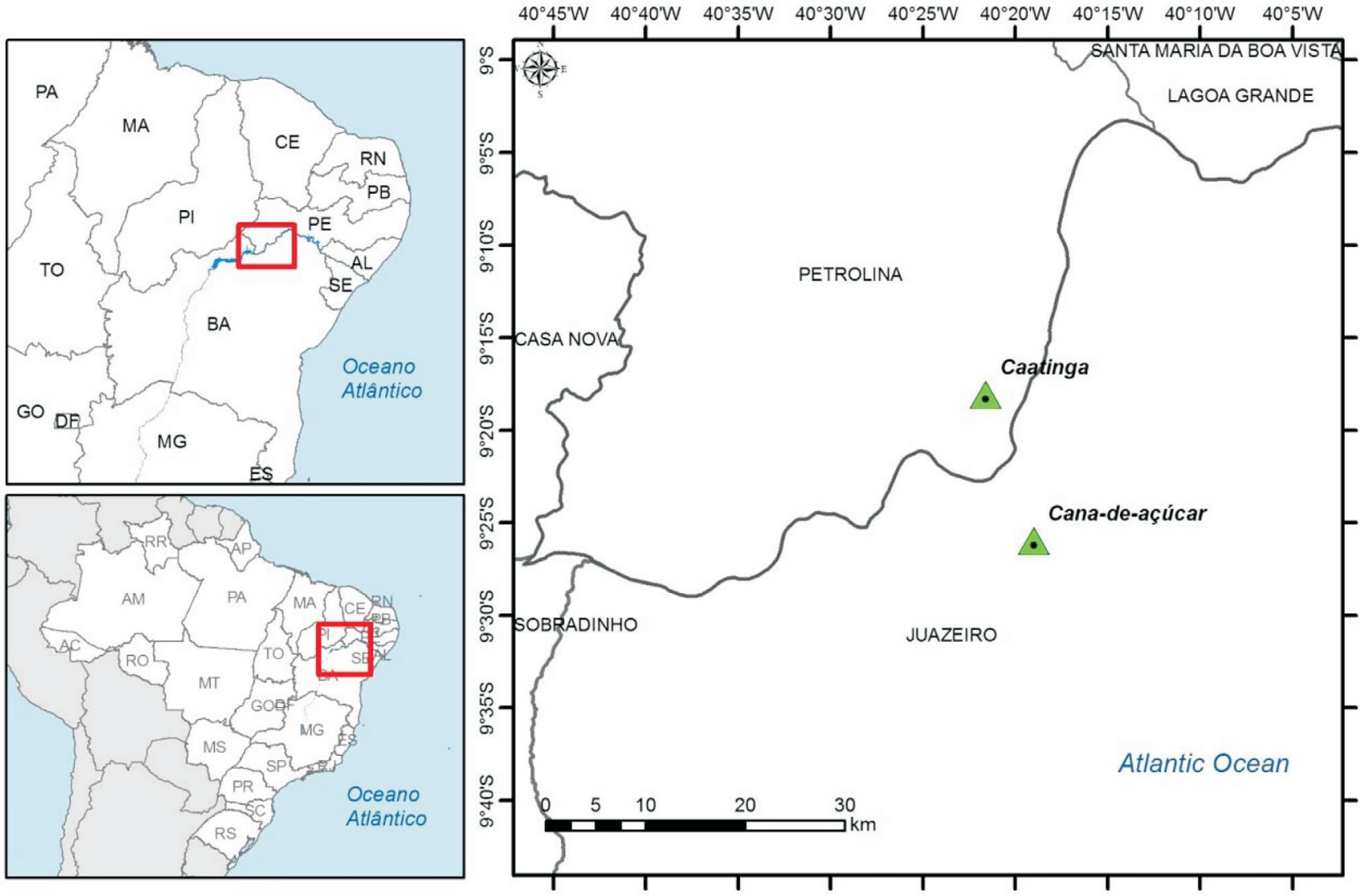

Figura 1 - Localização geográfica das torres micrometeorológicas instaladas áreas experimentais no Submédio do Vale São Francisco. 
$26,4{ }^{\circ} \mathrm{C}$, umidade relativa do ar média de $62 \%$ e precipitação anual de $520 \mathrm{~mm}$ (Embrapa Semiárido, 2015).

O sítio de caatinga preservada se encontra no município de Petrolina-PE, em áreas da Embrapa Semiárido ( $09^{\circ} 05^{\prime} \mathrm{S}$; $40^{\circ} 19^{\prime} \mathrm{O}$; $350 \mathrm{~m}$ de altitude), ocupando $600 \mathrm{ha}$, preservada há mais de 35 anos, solo do tipo Argissolo Amarelo Eutrófico, composta por espécies de porte arbóreo-arbustivo, caráter espinhento, hiperxerófila, caducifólias, com folhas pequenas e altura variando de 4 a $7 \mathrm{~m}$.

Por sua vez, o cultivo agrícola de cana-de-açúcar (Saccharum officinarum spp.) foi conduzido no município de Juazeiro-BA, na empresa Agroindústria do Vale São Francisco (09 $26^{\prime} \mathrm{S}$; 40¹9' O; $396 \mathrm{~m}$ de altitude). A área plantada foi de 13,97 ha, solo do tipo Vertissolo, $3^{\circ}$ ciclo produtivo, variedade VAT 90-212, fileiras em espaçamento duplo de $1,3 \mathrm{~m} \times 1,0 \mathrm{~m}$, irrigada por gotejamento subsuperficial. As lâminas de irrigação foram calculadas com base na evapotranspiração de referência, por meio do método de Penman-Monteith parametrizado no boletim 56 da FAO (Allen et al., 1998), considerando dados meteorológicos coletados de uma estação agrometeorológica, situada próxima a área experimental.

Em cada sítio experimental foi instalada uma torre micrometeorológica equipada com sensores eletrônicos para quantificação dos componentes do balanço de radiação, energia e demais parâmetros ambientais. Na caatinga, a torre era de $16 \mathrm{~m}$ de altura, com dois sistemas de medidas, um em baixa frequência para medições dos componentes do balanço de radiação (CNR1 Net radiometer, Kip \& Zonen, B.V. Delft Netherlands), temperatura e umidade relativa do ar (HMP45, Campbell Scientific, Logan, Utah, USA) e precipitação (CS700-L, Hydrological Services Rain Gage, Liverpool, Austrália). No solo foi instalado um termistor (CS107, Campbell Scientific, INC, Logan, Utah, USA) para medição da temperatura do solo, enquanto o fluxo de calor no solo foi medido por meio de fluxímetro (HFT3-REBS, sCampbell Scientific, INC, Logan, Utah, USA).

Já o sistema de alta frequência realizava medidas em $10 \mathrm{~Hz}$ e era composto por um anemômetro sônico tridimensional (CSAT-3, Campbell Scientific, Inc., Logan, UT, EUA) para medição das flutuações da velocidade do vento e da temperatura do ar, além de um analisador de gás ao infravermelho (LI-7500, LI-COR, Inc. Liconln, NE, EUA) para obtenção da concentração de vapor d'agua. Os dados da área de caatinga foram tabulados segundo a metodologia descrita em Souza et al. (2015a), em escala de $30 \mathrm{~min}$, utilizando o software Alteddy, considerando as equações (1) e (2):

$$
\begin{aligned}
& H=\rho_{a} C_{c} \overline{w^{\prime} T^{\prime}} \\
& L E=\overline{L w^{\prime} q^{\prime}}
\end{aligned}
$$

em que $L E$ - fluxo de calor latente $\left(\mathrm{W} \mathrm{m}^{-2}\right) ; H$ - fluxo de calor sensível $\left(\mathrm{W} \mathrm{m}^{-2}\right) ; L$ - calor latente de vaporização
$\left(\mathrm{KJ} \mathrm{kg}^{-1}\right) ; w^{\prime}$ - desvio instantâneo da velocidade do vento em relação ao valor médio $\left(\mathrm{m} \mathrm{s}^{-1}\right) ; q^{\prime}$ - desvio instantâneo da densidade vapor d'água em relação ao valor médio $\left(\mathrm{kg} \mathrm{m}^{-3}\right) ; \rho_{a}$ - densidade do ar $\left(\mathrm{kg} \mathrm{m}^{-3}\right) ; C_{p}$ - calor específico do ar à pressão constante $\left(\mathrm{KJ} \mathrm{kg}^{-1}\right) ; q$ - umidade específica $\left(\mathrm{g} \mathrm{g}^{-1}\right) ; T$ - temperatura do ar $(\mathrm{K})$.

Por sua vez, no sítio da cana-de-açúcar a torre era de $8 \mathrm{~m}$, equipada com os mesmos sensores de baixa frequência, descritos para o sítio caatinga. Ademais, foram instalados três conjuntos psicrométricos ventilados, para medir temperatura do ar em bulbo seco e em bulbo úmido, posicionados a 0,$50 ; 2,0$ e $3,5 \mathrm{~m}$ sobre a cultura, realocados conforme o crescimento das plantas.

O saldo de radiação $(R n)$ e fluxo de calor no solo $(G)$ foram medidos, enquanto o $L E$ e $H$ foram estimados utilizando o método do balanço de energia da razão de Bowen $(\beta)$, conforme as equações (3) e (4).

$$
\begin{aligned}
& L E=\frac{R n-G}{1+\beta} \\
& H=\frac{\beta(R n-G)}{1+\beta}
\end{aligned}
$$

em que $\beta$ é a razão de Bowen (adimensional).

A razão de Bowen ( $\beta$ ) foi determinada por meio da expressão psicrométrica conforme Righi et al. (2007), apresentada na equação (5).

$$
\beta=\left(\frac{\Delta+\gamma}{\gamma} \frac{t_{b u 1}-t_{b s 1}}{t_{b u 2}-t_{b s 2}}-1\right)
$$

em que $\Delta$ - declividade da curva de pressão de saturação do vapor d'água $\left(\mathrm{kPa}{ }^{\circ} \mathrm{C}^{-1}\right) ; \gamma$ - constante psicrométrica $\left(\mathrm{kPa}^{\circ} \mathrm{C}^{-1}\right) ; t_{b u}$ - temperatura do bulbo úmido no nível $\mathrm{Z} 1 \mathrm{e}$ $\mathrm{Z} 2\left({ }^{\circ} \mathrm{C}\right) ; t_{b s}$ - temperatura do bulbo seco no nível Z1 e $\mathrm{Z} 2\left({ }^{\circ} \mathrm{C}\right)$.

Para calcular os erros intrínsecos na determinação dos valores de $\beta$ e, por conseguinte, LE, aplicou-se os critérios definidos na metodologia de Perez et al. (1999), e a consistência física dos dados foi verificada conforme procedimentos citados no trabalho de Silva et al. (2011).

Para determinação do índice de cobertura vegetal (ICV), utilizou-se o método não destrutivo da interceptação da luz, realizando medidas de radiação fotossinteticamente ativa acima e abaixo do dossel, por meio do sensor portátil ceptômetro (AccuPAR, LP-80, Decagon Devices, Pullman, USA), a cada 15 dias em subparcelas representativas de cada área de estudo. O monitoramento da umidade do solo foi realizado por meio do sensor CS658 HydroSence (Campbell Scientific, INC, Logan, Utah, USA), efetuando leituras a cada 15 dias em ambas as áreas.

Os dados de 30 minutos dos componentes do balanço de radiação e energia foram integrados em escala diária, agrupados em cinco períodos, considerando a precipitação ( $>1 \mathrm{~mm})$ e o número de dias com chuva, conforme os 
seguintes limites: período seco: chuva acumulada $<20 \mathrm{~mm}$ com menos de cinco dias chuvosos, nos 30 dias anteriores e 30 dias sucessivos; chuvoso: $>20 \mathrm{~mm}$, com pelos menos cinco dias chuvosos, nos 30 dias anteriores e 30 dias sucessivos; e transição: condições intermediárias aos casos anteriores.

Assim, os períodos de avaliação foram definidos da seguinte forma: período seco/2015 (01/06/2015 a 06/11/2015); período de transição seco/chuvoso (07/11/2015 a 07/01/2015); período chuvoso (08/01/2016 a 31/01/2016); período de transição chuvoso/seco (01/02/2016 a 25/05/2016) e período seco /2016 (26/05/16 a $06 / 11 / 2016$ ).

Os dados foram submetidos à análise estatística não paramétrica com comparação da variabilidade dos fluxos e das variáveis ambientais entre os cinco períodos da mesma superfície, utilizando os testes de Kruskal-Wallis e Dunn (KW-D, $\alpha=0,05$ ). Quando o intuito foi realizar a comparação entre as duas superfícies no mesmo período, aplicou-se o teste de Pettitt-Mann-Whitney (MW, $\alpha=0,05$ ); todas as análises foram feitas com auxílio do software XLSTAT v. 2015 (Pingale et al., 2016).

\section{Resultados e Discussão}

A sazonalidade e a magnitude dos balanços de radiação e de energia foram diferentes entre as superfícies estudadas $(\mathrm{p}<0,05)$ em função da época do ano, regime hídrico e características inerentes a cada tipo de vegetação $(\mathrm{p}<0,05)$.

No padrão sazonal dos componentes do balanço de radiação da caatinga, percebeu-se que, a $\mathrm{K} \downarrow$ foi maior no período transição seco/chuvoso (KW-D, $\mathrm{p}<0,05$, Tabela 1), por causa da maior quantidade de dias de céu claro e a menor inclinação dos raios solares para a região (declinação solar em torno da latitude local). No período seguinte, com o início dos eventos de chuva, houve redução da $\mathrm{K} \downarrow$ devido a maior nebulosidade (Tabela 1). Variações semelhantes na $\mathrm{K} \downarrow$ foram relatadas nesta mesma região de es- tudo por Silva et al. (2011), Santos et al. (2012) e Souza et al. (2015b), analisando a sazonalidade do balanço de radiação. Chen et al. (2016) afirmaram que essa variável é influenciada, sobretudo pela localização e presença de nuvens.

Em relação ao componente $\mathrm{K} \uparrow$ da caatinga, o período de transição seco/chuvoso também apresentou maior refletividade da radiação (KW-D, p $<0,05$, Tabela 1), por causa da alta K $\downarrow$ e baixo ICV (média de $1,9 \mathrm{~m}^{2} \mathrm{~m}^{-2}$, Fig. 2). A caatinga é caducifólia, logo perde totalmente as folhas, ficando apenas galhos acinzentados, quando a umidade no solo é reduzida (Tabela 2). Resultados análogos foram verificados por Souza et al. (2015b) nos meses correspondentes ao período transição seco/chuvoso, durante o ano de 2012, no mesmo tipo de vegetação e ambiente. O período seco/chuvoso foi marcado por longa estiagem, resultando em diminuição foliar, diferente do período de transição chuvoso/seco, quando há persistência das folhas nas plantas (Fig. 2).

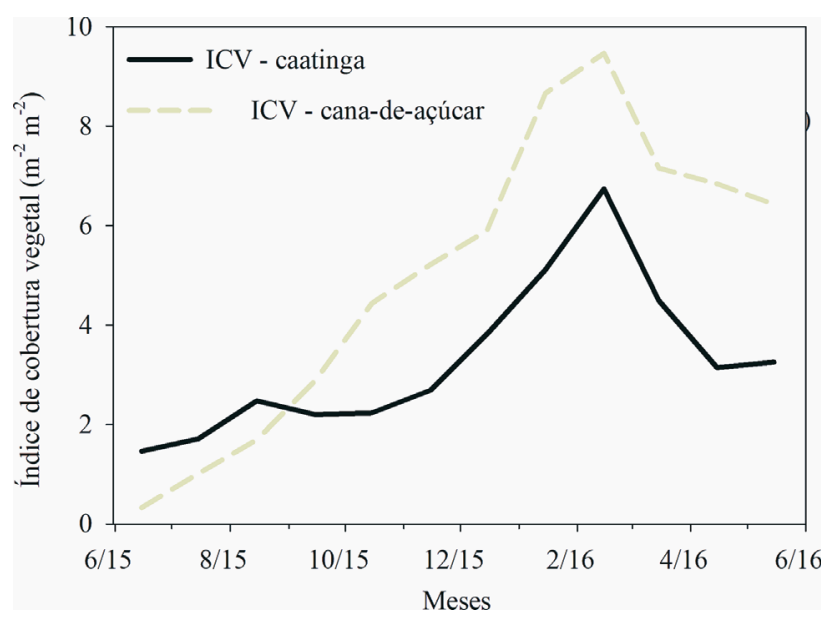

Figura 2 - Índice de cobertura vegetal (ICV) da caatinga preservada e cana-de-açúcar irrigada no Submédio do Vale do São Francisco, Semiárido brasileiro.

Tabela 1 - Valores médios dos componentes do balanço de radiação: radiação solar global $(\mathrm{K} \downarrow)$, radiação solar refletida (K个), radiação solar emitida da superfície $(\mathrm{L} \uparrow)$ e radiação solar atmosférica $(\mathrm{L} \downarrow)$, em área de caatinga preservada e cana-de-açúcar irrigada no Submédio do Vale São Francisco, Semiárido brasileiro

\begin{tabular}{|c|c|c|c|c|c|c|c|c|}
\hline \multirow[t]{2}{*}{ Período } & \multicolumn{2}{|c|}{$\mathrm{K} \downarrow\left(\mathrm{MJ} \mathrm{m}^{-2} \mathrm{dia}^{-1}\right)$} & \multicolumn{2}{|c|}{$\mathrm{K} \uparrow\left(\mathrm{MJ} \mathrm{m}^{-2} \mathrm{dia}^{-1}\right)$} & \multicolumn{2}{|c|}{$\mathrm{L} \uparrow\left(\mathrm{MJ} \mathrm{m}^{-2} \mathrm{dia}^{-1}\right)$} & \multicolumn{2}{|c|}{$\mathrm{L} \downarrow\left(\mathrm{MJ} \mathrm{m}^{-2} \mathrm{dia}^{-1}\right)$} \\
\hline & Caatinga & Cana & Caatinga & Cana & Caatinga & Cana & Caatinga & Cana \\
\hline Seco/2015 & $21,4 \mathrm{Ab}$ & $21,1 \mathrm{Ab}$ & $2,3 \mathrm{Ab}$ & $2,8 \mathrm{Ac}$ & $39,1 \mathrm{Ab}$ & $39,1 \mathrm{Ab}$ & $33,4 \mathrm{Ab}$ & $32,7 \mathrm{Bc}$ \\
\hline Seco/chuvoso & $26,2 \mathrm{Aa}$ & $25,7 \mathrm{Aa}$ & $3,1 \mathrm{Ba}$ & 4,5 Aa & $42,0 \mathrm{Aa}$ & $40,0 \mathrm{Ba}$ & $34,7 \mathrm{Aab}$ & $34,2 \mathrm{Ab}$ \\
\hline Chuvoso & $18,6 \mathrm{Ab}$ & $17,8 \mathrm{Ab}$ & $2,3 \mathrm{Bb}$ & $3,1 \mathrm{Ac}$ & $39,9 \mathrm{Ab}$ & $39,0 \mathrm{Ab}$ & $36,7 \mathrm{Aa}$ & $35,8 \mathrm{Aa}$ \\
\hline Chuvoso/seco & $22,2 \mathrm{Ab}$ & $22,5 \mathrm{Ab}$ & $2,8 \mathrm{Bb}$ & 4,1 Aab & $38,5 \mathrm{Bb}$ & $39,2 \mathrm{Ab}$ & $33,8 \mathrm{Ab}$ & $33,9 \mathrm{Ab}$ \\
\hline Seco/2016 & $15,3 \mathrm{Ab}$ & $16,4 \mathrm{Ab}$ & $1,9 \mathrm{Bb}$ & $3,2 \mathrm{Abc}$ & $39,3 \mathrm{Ab}$ & $38,4 \mathrm{Bb}$ & $36,0 \mathrm{Aab}$ & $34,3 \mathrm{Aab}$ \\
\hline
\end{tabular}

Médias seguidas pela mesma letra maiúscula na horizontal não diferem estatisticamente entre si ao nível de significância $(p<0,05)$ pelo teste não paramétrico de Kruskal-Wallis; Médias seguidas pela mesma letra minúscula na vertical não diferem estatisticamente entre si ao nível de significância $(\mathrm{p}<0,05)$ pelo teste não paramétrico de Mann-Whitney. 
Tabela 2 - Precipitação (Prec.), irrigação (Irr.) e média da temperatura do solo (Ts) e umidade do solo (Us), em área de caatinga preservada e cana-de-açúcar irrigada no Submédio do Vale São Francisco, Semiárido brasileiro

\begin{tabular}{|c|c|c|c|c|c|c|c|}
\hline \multirow[t]{2}{*}{ Períodos } & \multicolumn{2}{|c|}{ Prec. } & \multirow{2}{*}{$\begin{array}{c}\text { Irr. } \\
\text { Cana }\end{array}$} & \multicolumn{2}{|c|}{ Ts $\left({ }^{\circ} \mathrm{C}\right)$} & \multicolumn{2}{|c|}{ Us (\%) } \\
\hline & Caatinga & Cana & & Caatinga & Cana & Caatinga & Cana \\
\hline $\mathrm{Seco} / 2015$ & 11 & 17 & 753 & $31,4 \mathrm{Ab}$ & $26,4 \mathrm{Bb}$ & $1,4 \mathrm{Bb}$ & $47,0 \mathrm{Ab}$ \\
\hline Seco/Chuvoso & 107 & 51 & 361 & $36,4 \mathrm{Aa}$ & $28,8 \mathrm{Ba}$ & 5,3 $\mathrm{Bab}$ & $44,3 \mathrm{Ab}$ \\
\hline Chuvoso & 214 & 254 & 15 & $28,2 \mathrm{Ac}$ & $25,5 \mathrm{Bb}$ & $18,2 \mathrm{Ba}$ & $50,3 \mathrm{Aa}$ \\
\hline Chuvoso/Seco & 68 & 45 & 489 & $30,1 \mathrm{Ab}$ & $25,3 \mathrm{Bb}$ & $11,6 \mathrm{Bb}$ & $48,8 \mathrm{Ab}$ \\
\hline $\mathrm{Seco} / 2016$ & 5 & 0 & 0 & $26,7 \mathrm{Ac}$ & $24,4 \mathrm{Ab}$ & 5,2 Bab & $42,4 \mathrm{Ab}$ \\
\hline
\end{tabular}

Médias seguidas pela mesma letra maiúscula na horizontal não diferem estatisticamente entre si ao nível de significância $(p<0,05)$ pelo teste não paramétrico de Kruskal-Wallis; Médias seguidas pela mesma letra minúscula na vertical não diferem estatisticamente entre si ao nível de significância $(\mathrm{p}<0,05)$ pelo teste não paramétrico de Mann-Whitney.

No mesmo período seco/chuvoso, a radiação de ondas longas emitida pela superfície (L个) foi mais elevada quando comparada aos demais períodos (KW-D, $\mathrm{p}<0,05$, Tabela 1). A alta incidência de $\mathrm{K} \downarrow$ aumentou o ganho de energia e, por conseguinte, a temperatura do solo (Tabela 2). A baixa nebulosidade durante esse período reduziu a radiação de ondas longas reemitida da atmosfera para a superfície (L $\downarrow$ ) (Tabela 1), apresentando-se maior no período chuvoso.

Analisando o balanço de radiação na área de canade-açúcar, percebeu-se que os padrões da sazonalidade da $\mathrm{K} \downarrow, \mathrm{L} \uparrow$ e $\mathrm{L} \downarrow$ foram semelhantes ao da caatinga. A única mudança ao longo do tempo foi constatada para a $\mathrm{K} \uparrow$, que depende da $\mathrm{K} \downarrow$ e do crescimento da cultura. De acordo com André et al. (2010), os valores de $\mathrm{K} \uparrow$ variam conforme o crescimento da cultura, em razão da cobertura do solo, contribuindo para aumento do albedo e, alterando o balanço de radiação.

Comparando os componentes do balanço de radiação entre essas superfícies, a $K \downarrow$ não apresentou diferença entre os sítios estudados (MW, p >0,05), já que, as áreas experimentais são planas e próximas entre si $(<70 \mathrm{~km}$ em linha reta), ou seja, a incidência de energia que atinge as superfícies é a mesma (Tabela 1). Com exceção do período seco/2015, quando o ICV das duas superfícies foi baixo
(Tabela 1), nos demais períodos a $\mathrm{K} \uparrow$ da cana-de-açúcar foi superior ao da caatinga. Houve incremento de $1,1 \mathrm{MJ} \mathrm{m}^{-2}$ dia $^{-1}$, em média, na $\mathrm{K} \uparrow$, que corresponde a $42 \%$ de aumento na reflectância da superfície ao se mudar caatinga preservada por cana-de-açúcar. Von Randow et al. (2004) e Souza et al. (2013) citaram aumento de $58 \%$ e mais de $100 \%$ na radiação refletida ao substituir vegetação nativa por pastagens e soja, nessa ordem, na região Amazônia.

$\mathrm{O}$ aumento do ICV da cana-de-açúcar durante o período de transição seco/chuvoso resultou em menor emissão de $\mathrm{L} \uparrow(\mathrm{MW}, \mathrm{p}<0,05)$, por causa da maior reflexão da superfície (Tabela 1), ou seja, neste período, uma menor quantidade de energia foi absorvida pela cultura, quando comparado à caatinga. Com o aumento do ICV da cana-deaçúcar, a $\mathrm{L} \uparrow$ tendeu a ser maior durante o período chuvoso/seco (MW, $\mathrm{p}<0,05$ ). A partir deste momento, o $\mathrm{L} \uparrow$ foi inferior ao da caatinga devido ao tombamento da cultura no final do ciclo, o que promoveu a abertura do dossel e favoreceu a retenção de radiação.

Em termos de balanço de energia, a variação do Rn na caatinga foi idêntica ao da $\mathrm{K} \downarrow$, com maior magnitude ocorrendo no período de transição seco/chuvoso (KW-D, $\mathrm{p}<0,05$, Tabela 3). Neste período, a alta $\mathrm{K} \downarrow$ associada à umidade do solo ainda baixa resultou em maiores valores de $\mathrm{H}$ e de $\mathrm{G}$. O LE foi maior nos períodos chuvoso e

Tabela 3 - Valores médios dos componentes do balanço de energia: saldo de radiação $(R n)$, fluxo de calor sensível $(H)$, latente $(L E)$ e de calor no solo $(G)$, em área de caatinga preservada e cana-de-açúcar irrigada no Submédio do Vale São Francisco, Semiárido brasileiro

\begin{tabular}{|c|c|c|c|c|c|c|c|c|}
\hline \multirow[t]{2}{*}{ Período } & \multicolumn{2}{|c|}{$R n\left(\mathrm{MJ} \mathrm{m}^{-2} \mathrm{dia}^{-1}\right)$} & \multicolumn{2}{|c|}{$H\left(\mathrm{MJ} \mathrm{m}^{-2} \mathrm{dia}^{-1}\right)$} & \multicolumn{2}{|c|}{$L E\left(\mathrm{MJ} \mathrm{m}^{-2} \mathrm{dia}^{-1}\right)$} & \multicolumn{2}{|c|}{$G\left(\mathrm{MJ} \mathrm{m}^{-2} \mathrm{dia}^{-1}\right)$} \\
\hline & Caatinga & Cana & Caatinga & Cana & Caatinga & Cana & Caatinga & Cana \\
\hline Seco/2015 & $14,3 \mathrm{Ab}$ & $14,3 \mathrm{Ab}$ & $11,6 \mathrm{Ab}$ & 2,3 Ba & $2,6 \mathrm{Bb}$ & $11,1 \mathrm{Ab}$ & $0,1 \mathrm{Bab}$ & $0,8 \mathrm{Aa}$ \\
\hline Seco/chuvoso & $18,2 \mathrm{Aa}$ & $17,5 \mathrm{Ba}$ & $14,2 \mathrm{Aa}$ & $0,8 \mathrm{Bc}$ & $3,7 \mathrm{Bb}$ & $16,4 \mathrm{Aa}$ & $0,3 \mathrm{Aa}$ & $0,4 \mathrm{Aa}$ \\
\hline Chuvoso & $14,0 \mathrm{Ab}$ & $13,7 \mathrm{Ab}$ & $5,9 \mathrm{Ac}$ & $1,0 \mathrm{Bbc}$ & $8,3 \mathrm{Ba}$ & $12,8 \mathrm{Ab}$ & $-0,2 \mathrm{Ab}$ & $-0,2 \mathrm{Ab}$ \\
\hline Chuvoso/seco & $16,6 \mathrm{Ab}$ & $14,8 \mathrm{Bb}$ & $10,2 \mathrm{Ac}$ & $2,7 \mathrm{Ba}$ & $6,1 \mathrm{Ba}$ & $12,3 \mathrm{Ab}$ & $0,2 \mathrm{Ab}$ & $-0,2 \mathrm{Bb}$ \\
\hline Seco/2016 & $11,4 \mathrm{Ab}$ & $11,1 \mathrm{Ab}$ & $7,1 \mathrm{Ac}$ & $1,9 \mathrm{Bab}$ & $4,8 \mathrm{Bb}$ & $9,4 \mathrm{Ab}$ & $-0,4 \mathrm{Ab}$ & $-0,3 \mathrm{Ab}$ \\
\hline
\end{tabular}

Médias seguidas pela mesma letra maiúscula na horizontal não diferem estatisticamente entre si ao nível de significância $(\mathrm{p}<0,05)$ pelo teste não paramétrico de Kruskal-Wallis; Médias seguidas pela mesma letra minúscula na vertical não diferem estatisticamente entre si ao nível de significância $(\mathrm{p}<0,05)$ pelo teste não paramétrico de Mann-Whitney. 
Tabela 4 - Valores médios das partições de energia disponível para os fluxos de calor latente $(L E / R n)$, sensível $(H / R n)$ e calor no solo $(G / R n)$ em área de caatinga preservada e cana-de-açúcar irrigada no Submédio do Vale São Francisco, Semiárido brasileiro

\begin{tabular}{|c|c|c|c|c|c|c|}
\hline \multirow[t]{2}{*}{ Períodos } & \multicolumn{2}{|c|}{$L E / R n$} & \multicolumn{2}{|c|}{$H / R n$} & \multicolumn{2}{|c|}{$G / R n$} \\
\hline & Caatinga & Cana & Caatinga & Cana & Caatinga & Cana \\
\hline Média & $0,34 \mathrm{~B}$ & $0,85 \mathrm{~A}$ & $0,64 \mathrm{~A}$ & $0,12 \mathrm{~B}$ & $0,02 \mathrm{~A}$ & $0,03 \mathrm{~A}$ \\
\hline
\end{tabular}

Médias seguidas pela mesma letra maiúscula na horizontal não diferem estatisticamente entre si ao nível de significância $(p<0,05)$ pelo teste não paramétrico de Kruskal-Wallis; Médias seguidas pela mesma letra minúscula na vertical não diferem estatisticamente entre si ao nível de significância $(\mathrm{p}<0,05)$ pelo teste não paramétrico de Mann-Whitney.

chuvoso/seco, quando houve maior umidade do solo. De acordo com Sun et al. (2010), a variação das trocas de energia entre a superfície e a atmosfera em regiões com limitação hídrica é explicada pelo ICV, precipitação e demanda atmosférica do local. Já Malhi et al. (2002) citam que períodos prolongados de seca modificam os padrões de energia, em função da umidade no solo e regulação da vegetação.

Na cana-de-açúcar, o Rn seguiu o mesmo padrão da caatinga, ao passo que os valores de $\mathrm{H}$ foram superiores nos períodos seco/2015, chuvoso/seco e seco/2016 (KW-D, $\mathrm{p}<0,05$, Tabela 3), devido a maior exposição do solo, suspensão da irrigação e tombamento da cultura, nessa ordem. O LE apresentou maiores magnitudes no período seco/chuvoso quando, associada à aplicação das lâminas de irrigação, houve alta intensidade de $\mathrm{K} \downarrow$. O G foi superior entre os períodos seco/2015 e seco/chuvoso, quando o ICV ainda era reduzido.

Comparando as duas superfícies, o $\mathrm{Rn}$ da cana-deaçúcar foi, em média, 1,03 $\mathrm{MJ} \mathrm{m}^{-2} \mathrm{dia}^{-1}$ menor do que a caatinga (MW, p <0,05, Tabela 3 ). Nesta última vegetação, a menor radiação refletida e a maior emissão de radiação de ondas longas na maioria do ano, justificam a maior disponibilidade de energia no sistema em relação à cana-de-açúcar. Fausto et al. (2016) citaram alterações do Rn como reflexo dos vários tipos de uso e ocupação do solo. Pongratz et al. (2006) e Cunha et al. (2013) afirmaram que a mudança do uso do solo causa alterações significativas nos atributos biofísicos da superfície, alterando a rugosidade e as propriedades aerodinâmicas e, por conseguinte, os fluxos de energia e massa.

As magnitudes do $\mathrm{H}$ na caatinga, como era de se esperar, foram maiores durante todos os períodos em relação a cana-de-açúcar (MW, p < 0,05, Tabela 3), devido à baixa umidade do solo. Sob esta condição, o fechamento estomático pelas plantas é mais frequente, logo, maior parte da energia disponível é destinada ao aquecimento do ar. Já o LE foi superior na área de cana-de-açúcar em decorrência do regime de água durante todo o ciclo (irrigação mais precipitação igual a $1984 \mathrm{~mm}$ ). O G foi maior na cana-deaçúcar no período seco/2015, quando o ICV era baixo, inferior ao da caatinga. Enquanto foi menor na cana-de-açúcar no período chuvoso/seco, quando a cultura atingiu o máximo ICV. O H da caatinga foi $82 \%$ maior do que no cultivo irrigado e o LE da cana-de-açúcar foi 59\% maior do que a vegetação nativa. Alterações nos componentes do balanço de energia entre a caatinga e cultivos irrigados também foram relatados por Teixeira et al. (2008).

De modo geral, na caatinga as partições de energia para o LE, $\mathrm{H}$ e $\mathrm{G}$ foram $34 \%, 64 \%$ e $2 \%$, nessa ordem, indicando maior uso de energia para o aquecimento do ar (MW, p < 0,05, Tabela 4). No cultivo irrigado de cana-deaçúcar, a magnitude destinada ao LE foi de $85 \%$, enquanto $12 \%$ para o $\mathrm{H}$ e $3 \%$ para o $\mathrm{G}$; estes valores são significativamente diferentes aos observados na caatinga (MW, $\mathrm{p}<0,05)$. Teixeira et al. (2008) encontraram, entre os anos de 2004 e 2005, na mesma região de estudo desta pesquisa, magnitudes de $79 \%, 89 \%$ e $77 \%$ da partição do Rn para o LE em cultivos irrigados de uva de vinha, uva de mesa e manga, respectivamente; enquanto para a caatinga foi de $33 \%$. Para o $\mathrm{H}$, estes mesmos autores citam 20\%, 13\%, $14 \%$ e $56 \%$, nessa ordem. Silva et al. (2011), para a canade-açúcar irrigada por sulco nesta mesma região, encontraram $81 \%, 16 \%$ e 3\% do Rn destinados para o LE, H e G.

\section{Conclusões}

As principais diferenças no balanço de radiação entre a caatinga e a cana-de-açúcar estão associadas ao albedo (superior no cultivo agrícola) e a emissão de radiação de ondas longas (maior na vegetação nativa), mas dependem das características de época do ano, índice de cobertura vegetal e de umidade do solo.

Os fluxos de calor sensível (H) e latente (LE) foram significativamente distintos entre as superfícies durante todo o ano, mesmo nos períodos de maior precipitação pluviométrica, sendo o $\mathrm{H}$ sempre maior na caatinga e o LE na cana-de-açúcar, independentemente, da época do ano.

A partição de energia na caatinga foi mais significativa para o aquecimento do ar, sendo em média $64 \%$ do saldo de radiação. Já no cultivo irrigado de cana-de-açúcar a partição foi mais para o processo de evapotranspiração, com média de $85 \%$ do saldo de radiação.

\section{Agradecimentos}

Ao CNPq (Processo n. 483223/2011-5) e FACEPE (Processo n. APQ-0062-1.07/15) pelo auxílio financeiro e a CAPES pela concessão da Bolsa de pós-graduação da primeira autora. 


\section{Referências}

ALLEN, R.G.; PEREIRA, L.S.; RAES, D.; SMITH, M. Crop evapotranspiration: guidelines for computing crop water requirements. 1ed. Rome: FAO, p. 326, 1998. (FAO. Irrigation and Drainage Paper, 56).

ANDRÉ, R.G.B.; MENDONÇA, J.C.; MARQUES, V.S.; PINHEIRO, F.M.A.; et al. Aspectos energéticos do desenvolvimento da cana-de-açúcar. Parte 1: Balanço de radiação e parâmetros derivados. Revista Brasileira de Meteorologia, São Paulo, v. 25, n. 3, p. 375-382, 2010.

CHAMBERS, J.Q.; ARTAXO, P. Biosphere-atmosphere interactions: Deforestation size influences rainfall. Nature Climate Change, New york, v. 7, n. 1, p. 175-176, 2017.

CHEN, X.; YU, Y.; CHEN, J.; ZHANG, T.; et al. Seasonal and interannual variation of radiation and energy fluxes over a rain-fed cropland in the semi-arid area of Loess Plateau, northwestern China. Atmospheric Research, Amsterdam, v. 176-177, n. 1, p. 240-253, 2016.

CUNHA, A.P.M.A.; ALVALÁ, R.C.S.; OLIVEIRA, G.S. Impactos das mudanças de cobertura vegetal nos processos de superfície na região semiárida do Brasil. Revista Brasileira de Meteorologia, São Paulo, v. 28, n. 2, p. 139-152, 2013.

DAVID, M.; WALKO, R.L.; AVISSAR, R. Effects of deforestation on spatio-temporal distributions of precipitation in South America. Journal of Climate, Boston, v. 24, n. 1, p. 2147-2163, 2011.

EMBRAPA SEMIÁRIDO. Médias anuais da Estação Agrometeorológica de Bebedouro. Petrolina, 2015. Disponível em: http:// www.cpatsa.embrapa.br:8080/servicos/dadosmet/ceb-anua 1.html. Acesso em: 16.03.2015.

FAUSTO, M.A.; ANGELINI, L.P.; MARQUES, H.O.; SILVA FILHO, A.S.; et al. Impacto da alteração do uso do solo no saldo de radiação no cerrado do sul de Mato Grosso. Revista Ambiente \& Água, v. 11, n. 2, p. 350-361 2016.

INSTITUTO BRASILEIRO DE GEOGRAFIA E ESTATÍSTICAS. Produção Agrícola Municipal 2014. www.sidra.ibge.gov.br/bda. Acesso em: 03 de agosto, 2016.

LU, S.; CHEN, S.; WILSKE, B.; SUN, G.; et al. Evapotranspiration and soil water relationships in a range of disturbed and undisturbed ecosystems in the semi-arid Inner Mongolia, China. Journal of Plant Ecology, Oxford, v. 4, n. 1-2, p. 49-60, 2011.

MALHI, Y.; PEGORARO, E.; NOBRE, A.D.; PEREIRA, M.G.P.; et al. The energy and water dynamics of a central Amazonian rain forest. Journal of Geophysical Research, Hoboken, v. 107, n. 20, p. 1-17, 2002.

MINISTÉRIO DO MEIO AMBIENTE. Caatinga. Disponível em: http://www.mma.gov.br/biomas/caatinga. Acesso em 20 de fevereiro de 2016.

OLIVEIRA, M.B.L.; SANTOS, A.J.B.; MANZI, A.O.; ALVALÁ, R.C.S.; et al. Trocas de energia e fluxo de carbono entre a vegetação de caatinga e atmosfera no nordeste brasileiro. Revista Brasileira de Meteorologia, São Paulo, v. 21, n. 3, p. 166-174, 2006.

PEREZ, P.J.; CASTELLVI, F.; MARTINEZ-COB, A. Assessment reliability of Bowen ratio method for partitioning flu- xes. Agricultural and Forest Meteorology, Amsterdam, v. 97, n. 1, p. 141-150, 1999.

PONGRATZ, J.; BOUNOUA, L.; DEFRIES, R.S.; MORTON, D.C.; et al. The impact of land cover change on surface energy and water balance in Mato Grosso, Brazil. Earth Interactions, Boston, v. 10, n. 1, p. 1-17, 2006.

PINGALE, S.M.; KHARE, D.; JAT, M.K.; ADAMOWSKI, J. Trend analysis of climatic variables in an arid and semi-arid region of the Ajmer District, Rajasthan, India. Journal Water and Land Development, India, v. 28, p. 3-18, 2016.

RIGHI, E.Z.; ANGELOCCI, L.R.; MARIN, F.R. Energy balance of a young drip-irrigated coffe crop in shoutheast Brazil: an analysis of errors and reliability of measurements by the Bowen ratio method. Revista Brasileira de Agrometeorologia, Sete Lagoas, v. 15, n. 3, p. 367-279, 2007.

SANTOS, S.A.; CORREIA, M.F.; ARAGÃO, M.R.S.; SILVA, P.K.O. Aspectos da variabilidade sazonal da radiação, fluxos de energia e $\mathrm{CO}_{2}$ em área de caatinga. Revista Brasileira de Geografia Física, Recife, v. 5, n. 4, p. 761-773, 2012.

SILVA, T.G.F.; MOURA, M.S.B.; ZOLNIER, S.; SOARES, J.M.; et al. Variação do balanço de radiação e energia da cana-de-açúcar irrigada no semiárido brasileiro. Revista Brasileira de Engenharia Agrícola e Ambiental, Campina Grande, v. 15, n. 2, p. 139-147, 2011.

SOUZA, P.J.O.P.; ROCHA, E.J.P.; RIBEIRO, A. Impactos do avanço da soja no balanço de radiação no leste da Amazônia. Acta Amazônica, Manaus, v. 43, n. 2, p. 169-178, 2013.

SOUZA, L.S.B.; MOURA, M.S.B.; SEDIYAMA, G.C.; SILVA, T.G.F. Balanço de energia e controle biofísico da evapotranspiração na Caatinga em condições de seca intensa. Revista Pesquisa Agropecuária Brasileira, Brasília, v. 50, n. 8, p. 627-636, 2015a.

SOUZA, L.S.B.; MOURA, M.S.B.; SEDIYAMA, G.C.; SILVA, T.G.F. Balanço de radiação em ecossistema de Caatinga preservada durante um ano de seca no Semiárido Pernambucano. Revista Brasileira de Geografia Física, Recife, v. 8 , n. 1, p. 41-55, 2015b.

SPRACKLEN, D.V.; ARNOLD, S.R.; TAYLOR, C.M. Observations of increased tropical rainfall preceded by air passage over forests. Nature, Brussels, v. 489, n. 1, p. 282-285, 2012.

SUN, G.; ALSTAD, K.; CHEN, J. CHEN, S.; et al. A general predictive model for estimating monthly ecosystem evapotranspiration. Ecohydrology, v. 4, n. 2, p. 245-255, 2010.

TEIXEIRA, A.H.C.; BASTIAANSSEN, W.G.M.; AHMAD, N.D.; MOURA, M.S.B.; et al. Analysis of energy fluxes and vegetation-atmosphere parameters in irrigated and natural ecosystems of semi-arid Brazil. Journal of Hydrology, Amsterdam, v. 362, n. 1-2, p. 110-127, 2008.

VON RANDOW, C.; MANZI, A. O.; KRUIJT, B.; OLIVEIRA, P.J.; et al. Comparative measurements and seasonal variations in energy and carbon exchange over forest and pasture in south west Amazonia. Theoretical and Applied Climatology, Wien, v. 78, n. 1, p. 5-26, 2004.

This is an Open Access article distributed under the terms of the Creative Commons Attribution Non-Commercial License which permits unrestricted non-commercial use, distribution, and reproduction in any medium provided the original work is properly cited. 